\title{
Muss der Patient nach einer Stimmlippenoperation wirklich schweigen?
}

\section{Voice Rest After Vocal Fold Surgery - What Can Be Recommended?}

Susanne Fleischer, Markus Hess

\author{
Die Empfehlungen, wie nach einer Stimmlippenoperation die Stimme zu schonen \\ oder zu gebrauchen sei, sind weltweit sehr unterschiedlich. Nicht selten werden \\ die Patienten zu mehrwöchiger kompletter Stimmruhe angehalten. Flüstern wird \\ im Allgemeinen nicht als Alternative angeboten. Aufgrund langjähriger Erfahrun- \\ gen halten die Autoren des Beitrags dagegen einen frühzeitigen, kontrollierten \\ Stimmgebrauch im Sinne einer Frührehabilitation für wichtig.
}

\section{LERNZIEL}

In diesem Beitrag wird an einer Literaturrecherche gezeigt, dass es zur Frage des Stimmgebrauchs nach einer Stimmlippenoperation bisher keine einheitliche Meinung gibt. Studien weisen darauf hin, dass komplette Stimmruhe nicht zu besseren Ergebnissen führt als Stimmschonung. Die Empfehlung zu Stimmruhe über mehr als 3 Tage ist umstritten. Möglicherweise führen Stimmübungen ab dem 3. postoperativen Tag zu besseren funktionellen Ergebnissen. Auch die Antwort auf die Frage, ob Flüstern schädlich ist, ist umstritten. Die Autoren sind der Meinung, dass lockeres und tonloses Flüstern direkt postoperativ nicht schädlich ist und erlaubt werden kann.

\section{Einleitung}

Die häufigste Indikation für Stimmlippenoperationen bei benignen Veränderungen ist das Ziel der Stimmverbesserung. Andererseits besteht bei jeder Stimmlippenoperation mit Eröffnen des Epithels das Risiko einer Stimmverschlechterung, wenn es im ungünstigen Falle zu einer ungewollten oder sogar übermäßigen Vernarbung innerhalb der Lamina propria mit Veränderungen der biomechanischen Eigenschaften der Stimmlippe kommt [1-4]. Die Behandlung von Stimmlippenvernarbungen ist sehr schwierig und es ist das primäre Ziel, den Heilungsprozess so zu beeinflussen, dass es nicht zu übermäßiger Narbenbildung kommt. Dabei spielt die Frage, ab wann die Stimmlippen wieder durch Stimmgebung belastet werden dürfen, eine wichtige Rolle.
Die Empfehlungen zum postoperativen Stimmgebrauch sind, Befragungen von Laryngologen zufolge, kontrovers [5-8]. Nicht selten werden die Patienten zu mehrwöchiger Stimmruhe angehalten und vom Flüstern wird ihnen im Allgemeinen dringend abgeraten. Den Patienten bleibt dann nur die schriftliche Kommunikation. Das führt zu einer erheblichen Einschränkung der Lebensqualität und hat eine begrenzte Compliance der Patienten zur Folge [9]. Dabei ist bisher nicht eindeutig nachgewiesen, dass Stimmruhe wirklich eine funktionell bessere Wundheilung nach sich zieht $[5,8,10]$.

In vielen Bereichen der Medizin hat sich inzwischen die Frührehabilitation mit kontrollierter Mobilisation und Bewegungsübungen bereits im frühen Heilungsstadium bewährt und eine längere Ruhigstellung hat sich als ungünstig erwiesen. Deshalb stellt sich die Frage, inwieweit auch für die Stimmlippen bei frühzeitiger postoperativer „Mobilisierung“, also bei frühzeitigem Stimmgebrauch, bessere funktionelle Ergebnisse zu erreichen sind und zu welchem Zeitpunkt welche Stimmübungen durchzuführen wären $[4,10,11]$.

\section{Phasen der Wundheilung und die mögliche Bedeutung für die Stimmlippenfunktion}

Heutzutage wird nach vielen orthopädischen Eingriffen eine Frührehabilitation empfohlen. Gewebe mit einer Bewegungsfunktion soll während der Wundheilungsphase frühzeitig mobilisiert werden, damit es „lernt“, wie es später funktionieren soll. 


\section{Merke}

Für die Stimmlippen würde Frühmobilisation bedeuten: Sie sollen longitudinal dehnbar sein, innerhalb der Schichtung der Lamina propria verschieblich sein und im Ganzen schwingen können.

Dieses Modell einer frühen Mobilisierung kann durch Untersuchungen des allgemeinen Wundheilungsprozesses erklärt werden, der sich in 3 verschiedene Phasen einteilen lässt $[1,3,10]$ :

- Inflammatorische (entzündliche) Phase: Diese erstreckt sich über die ersten 3 Tage.

- Proliferative Phase (Phase des schnellen Wachstums): In dieser Phase kommt es zum Einsprossen neuer Gefäße, zur Proliferation von Fibroblasten und zur Reepithelisierung. Diese Phase erstreckt sich über etwa einen Monat. Das Einwandern der Fibroblasten findet bereits nach $48-72 \mathrm{~h}$ statt. Die Fibroblasten spielen u.a. wegen der Produktion verschiedener extrazellulärer Substanzen eine wichtige Rolle. Es resultiert schließlich ein fibroblastenreiches, noch nicht differenziertes Granulationsgewebe.

- Maturationsphase (Reifungsphase oder auch Remodellierungsphase): Während dieser Phase, die nach etwa einem Monat beginnt und ein Jahr oder länger dauert, reift dieses fibroblastenreiche Gewebe weiter zu lockerem und später zu festerem fibrösem Bindegewebe, das mit der Zeit schrumpft und eine zellarme Narbe bildet.

Inwieweit dieser Ablauf auch der Wundheilung an den Stimmlippen entspricht, ist nicht eindeutig nachgewiesen, wesentliche Punkte scheinen jedoch vergleichbar zu sein [2, 3, 10-12]. Auch bei den Stimmlippen steht im Anfangsstadium die entzündliche Veränderung im Vordergrund. Nach einer kritischen Phase von 3 Tagen sind auch an den Stimmlippen Fibroblasten nachweisbar, die auf mechanische Reize reagieren und eine Schlüsselrolle im Wundheilungsprozess spielen sollen. Das Ausmaß der Veränderungen hängt natürlich auch von der Art des Eingriffs ab. Letztlich ist trotz der vorliegenden Studien vieles nicht bekannt und Fragen bezüglich möglicher Empfehlungen für einen optimalen Zeitplan der postoperativen Stimmgebung bleiben offen.

\section{Merke}

Sicher ist, dass eine extreme mechanische Belastung des Stimmlippengewebes postoperativ vermieden werden sollte $[5,11]$.

\section{Untersuchungen zum postoperativen Stimmgebrauch}

Es gibt bisher nur wenige Studien, in denen Ergebnisse von Patienten mit unterschiedlichem postoperativem Stimmgebrauch verglichen werden. In einer aktuellen Studie von Kaneko und Mitarbeitern wurden 31 Patienten nach phonochirurgischer Operation bei Leukoplakie, Stimmlippenzyste, Polyp, Reinke-Ödem oder Carcinoma in situ untersucht [1]. Sie sollten 3 bzw. 7 Tage postoperativ Stimmruhe einhalten und erhielten anschließend eine 6-wöchige Stimmtherapie mit Beratung zur Stimmhygiene und Einweisung in die sog. Tube-Phonation. Bei der Nachuntersuchung zeigten sich für die Gruppe mit der nur 3-tägigen Stimmruhe deutlich bessere Ergebnisse der Stimmfunktion als für die Gruppe mit der 7-tägigen Stimmruhe. Die Autoren erklären dies damit, dass eine angemessene mechanische Stimulation der Stimmlippen in der frühen Heilungsphase zu besseren funktionellen Ergebnissen führt. In einer Studie von anderen Autoren wurde die Stimmqualität nach 7 Tagen postoperativer Stimmruhe bzw. nach 7 Tagen postoperativer Stimmschonung untersucht. In den 2 Gruppen zeigten sich ähnliche Ergebnisse [13]. Eine andere Arbeitsgruppe verglich Patienten mit kompletter postoperativer Stimmruhe über 5 versus 10 Tage und fand bezüglich der Stimmbefunde keine signifikanten Unterschiede. Lediglich die maximale Tonhaltedauer (als nicht stimmqualitativer Parameter) war in der 2. Gruppe länger [14].

\section{Empfehlungen zur postoperativen Stimmruhe}

\section{Merke}

Man unterscheidet zwischen kompletter Stimmruhe, die völligem Schweigen entspricht, und Stimmschonung, unter der man im Allgemeinen versteht, dass nicht laut und nicht lange stimmhaft gesprochen wird, für die es aber keine eindeutige Definition gibt.

Zu bedenken ist in diesem Zusammenhang, dass beispielsweise das Vermeiden von Husten in den üblichen Empfehlungen oft nicht berücksichtigt wird. Husten und lautes Räuspern stellen jedoch eine deutlich gröBere Belastung für die Stimmlippen dar als leises Sprechen. High-Speed-Aufnahmen können sehr gut veranschaulichen, welch enorme Auslenkung das Stimmlippengewebe beim Husten und Räuspern erfährt und welch große negative Scherkräfte auf das Gewebe einwirken - im Gegensatz zur leisen Phonation oder zum Flüstern, bei denen keine Scherkräfte auftreten [15]. Eine absolute Immobilisierung der Stimmlippen ist praktisch nicht möglich, und zwar wegen der Bewegungen auch bei nicht phonatorischen laryngealen 


\section{FALLBEISPIEL}

Eine Opernsängerin stellte sich bei den Autoren des vorliegenden Beitrags mit akuter Stimmlippeneinblutung und Polypbildung vor ( $\triangleright$ Abb. 1). Nach Durchführung der Operation kam es zur regelrechten Wundheilung. Die Opernsängerin wurde zu frühzeitigen Stimmübungen mit allmählicher
Steigerung der Stimmbelastung und mit Anwendung von Semi-Occluded Vocal Tract Exercises beraten. Ihr geplanter Auftritt nach 3,5 Wochen in einer Hauptrolle bei der Premiere einer Wagner-Oper war anschließend problemlos möglich.
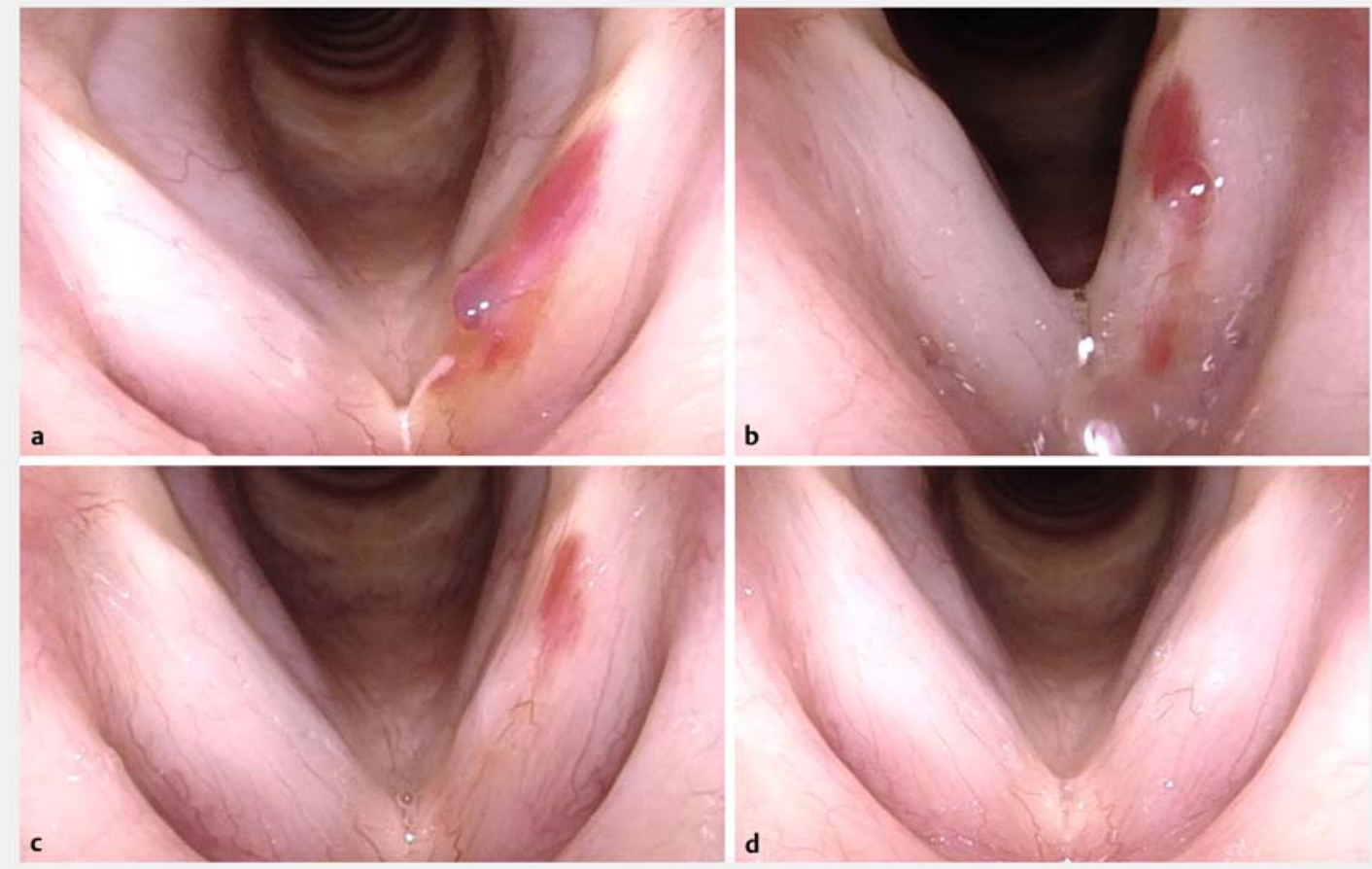

- Abb. 1 Opernsängerin mit akuter Stimmlippeneinblutung und Polypbildung. a Präoperativer Befund. b Befund direkt postoperativ, ca. $3 \mathrm{~h}$ nach der Operation in Vollnarkose. c Befund am 5. Tage postoperativ. $\mathbf{d}$ Befund am 14. Tage postoperativ.

Aktivitäten wie beispielsweise Schlucken, Pressen und Atmen [11].

Es gibt in der Literatur verschiedene Arbeiten mit Befragungen von Laryngologen zu Empfehlungen zum postoperativen Stimmgebrauch [5-8]. Die Empfehlungen variieren sehr und reichen bis hin zu 28 Tagen kompletter Stimmruhe. Am häufigsten wird die komplette Stimmruhe postoperativ über 7 Tage empfohlen. Stimmruhe bedeutet dabei in den meisten Befragungen, nicht zu sprechen, nicht zu flüstern und sich nicht zu räuspern. Zur Frage, ab welchem Zeitpunkt und in welchem Grad die Stimme wieder belastet werden kann, gibt es keine einheitlichen Angaben.

Die Empfehlungen, die die Autoren des vorliegenden Beitrags ihren Patienten geben, beruhen auf ihrer 30jährigen Erfahrung in der Phonochirurgie und der postoperativen Nachsorge [15]. Je nach Befund und durch- geführter Operation empfehlen sie nach einer Stimmlippenoperation mit Eröffnen des Epithels, die ersten 3 Tage postoperativ möglichst gar nicht bzw. sehr wenig und sehr leise zu sprechen. Anschließend kann die Stimmgebung langsam gesteigert werden. Etwa ab dem 5. Tag kann normal, aber ohne zu große Lautstärke gesprochen werden. Die Autoren raten ihren Patienten hingegen sehr dringend, als allerwichtigste Maßnahme Husten und Räuspern zu vermeiden. Außerdem fordern sie ihre Patienten auf, etwa ab dem 3.-5. Tag postoperativ mehrmals täglich mit geschlossenem Mund kurze und leise Glissando-Summübungen von tiefen bis zu sehr hohen Tönen durchzuführen, als eine Variante der Semi-Occluded Vocal Tract Exercises (SOVTE). Damit soll entsprechend dem Konzept einer frühen Rehabilitation das postoperativ neu einwachsende Gewebe in den Stimmlippen gedehnt und zum Schwingen gebracht werden. In besonderen Fällen empfehlen die Autoren im weiteren Verlauf zusätz- 
liche, logopädisch angeleitete Stimmübungen, beispielsweise mit gezielten und auf den Patienten abgestimmten Semi-Occluded Vocal Tract Exercises [16].

Weitere Empfehlungen ergeben sich aus der Art der durchgeführten Operation. Im Allgemeinen halten die Autoren eine Zunahme der Stimmbelastung nach 2 Wochen auf geschätzte $80 \%$ und nach 4 Wochen auf 100 \% für nicht schädlich. Ist aus anderen Gründen, beispielsweise bei einer zusätzlichen funktionellen Komponente der Stimmstörung, eine Stimmtherapie indiziert, kann diese meistens nach 2-3 Wochen beginnen. Da die Anforderungen bei Sängern sehr unterschiedlich sein können (z.B. Rock versus klassischer Gesang), beraten sie Sänger immer individuell, ab wann welche Belastung möglich ist, und bieten logopädische Therapie und gesangspädagogische Betreuung in Kombination an. Nach einer Stimmlippenaugmentation gelten andere Empfehlungen und das normale Sprechen wird in der Regel schon am nächsten Tag erlaubt und angeraten.

\section{Empfehlungen und Untersuchungen zum Flüstern}

In der Literatur lässt sich vielfach die Auffassung finden, dass Flüstern schädlich sei $[6,17]$. Obwohl allgemein anerkannt ist, dass es beim Flüstern nicht zu Schwingungen der Stimmlippen kommt, wird von manchen Autoren vermutet, dass Luftstromturbulenzen oder eine vermehrte muskuläre Anspannung die Stimmlippen schädigen. In den Empfehlungen, was Patienten postoperativ vermeiden sollen, wird Flüstern gleich nach Rufen und Singen genannt [6]. Andere Autoren fanden keinen möglichen schädlichen Mechanismus bei ihren Untersuchungen zum Flüstern [18].

In endoskopischen Untersuchungen an 100 Patienten sowie in High-Speed-Aufnahmen konnten die Autoren des vorliegenden Beitrags in einer vorangegangenen Studie zeigen, dass beim lockeren Flüstern keinerlei Bewegungen der Stimmlippen erkennbar sind. Hochfrequente Glottisschläge lassen sich nicht nachweisen und es kommt nicht zu einem festen Glottisschluss, sondern höchstens in einigen Fällen zu einem vibrationslosen Aneinanderliegen der anterioren Stimmlippen $[15,19]$. Messungen des subglottischen Druckes direkt endotracheal zeigen, dass dieser beim Flüstern deutlich niedriger als beim Sprechen ist und beim lockeren Flüstern deutlich niedriger als beim lauten Flüstern [20]. Diese Ergebnisse sprechen dafür, dass eine Schädigung des Stimmlippenepithels durch aerodynamische Drücke nicht zu erwarten ist und unangestrengtes Flüstern direkt postoperativ erlaubt werden kann $[15,18,19]$.

\section{FAZIT}

In der Literatur gibt es eine Studie, die darauf hinweist, dass Stimmübungen nach dem 3. postoperativen Tag möglicherweise zu besseren funktionellen Ergebnissen führen und Stimmruhe postoperativ nicht länger als 3 Tage erforderlich ist [1]. Dies deckt sich auch mit den jahrzehntelangen Erfahrungen der Autoren des vorliegenden Beitrags. Es lässt sich keine Studie finden, die nachweist, dass komplette Stimmruhe zu besseren Ergebnissen führt als Stimmschonung [5]. Eine extreme mechanische Belastung des Gewebes, wie sie beispielsweise beim Husten oder starken Räuspern durch Epithelverschiebungen mit großer Scherkraft in der Lamina propria auftreten kann, sollte postoperativ jedoch vermieden werden $[5,11,15]$.

\section{Schlüsselwörter}

Komplette Stimmruhe, Stimmschonung, Flüstern, Phonochirurgie, Frührehabilitation, Stimmübungen nach Phonochirurgie

\section{Interessenkonflikt}

Die Autorinnen/Autoren geben an, dass kein interessenkonflikt besteht

\section{Autorinnen/Autoren}

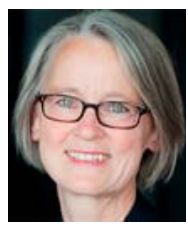

\section{Susanne Fleischer ${ }^{1,2}$}

Dr. med.; Fachärztin für Phoniatrie und Pädaudiologie sowie für HNO-Heilkunde, Kopfund Hals-Chirurgie. 1999-2014 Oberärztin an der Poliklinik für Hör-, Stimm- und Sprachheilkunde am Universitätsklinikum HamburgEppendorf. Schwerpunkt der klinischen Tätigkeit: videoendoskopische Diagnostik, u.a. Analyse von Stimmlippenveränderungen. 2014 Mitbegründerin der Deutschen Stimmklinik in Hamburg.

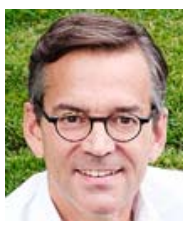

\section{Markus M. Hess}

Prof. Dr. med.; Facharzt für Phoniatrie und Pädaudiologie sowie für HNO-Heilkunde, Kopf- und Hals-Chirurgie. 1998-2014 Direktor der Klinik und Poliklinik für Hör-, Stimmund Sprachheilkunde am Universitätsklinikum Eppendorf. Forschungsschwerpunkte: Stimmphysiologie, Stimmlippenschwingungsanalyse und Stimmstörungen verschiedenster Ursachen, Behandlung von professionellen Sing- und Sprechstimmen. 2014 Mitbegründer der Deutschen Stimmklinik in Hamburg. 
Institute

1 Deutsche Stimmklinik, Hamburg

2 Klinik und Poliklinik für Hör-, Stimm- und Sprachheilkunde, Universitätsklinikum Hamburg-Eppendorf (UKE), Hamburg

\section{Korrespondenzadresse}

Susanne Fleischer

Deutsche Stimmklinik

Martinistraße 64

20251 Hamburg

E-Mail: fleischer@stimmklinik.de

\section{Literatur}

[1] Kaneko M, Shiromoto O, Fujiu-Kurachi M et al. Optimal duration for voice rest after vocal fold surgery: randomized controlled clinical study. J Voice 2017; 31: 97-103

[2] Hirano S. Current treatment of vocal fold scarring. Curr Opin Otolaryngol Head Neck Surg 2005; 13: 143-147

[3] Branski RC, Verdolini K, Rosen CA et al. Acute vocal fold wound healing in a rabbit. Ann Otol Rhinol Laryngol 2005; 114: 407-412

[4] Branski RC, Verdolini K, Sandulache V et al. Vocal fold wound healing: a review for clinicians. J Voice 2006; 20: 19-24

[5] Rihkanen H, Geneid A. Voice rest and sick leave after phonosurgical procedures: surveys among European laryngologists and phoniatricians. Eur Arch Otorhinolaryngol 2019; $276: 483-487$

[6] Coombs AC, Carswell AJ, Tierney PA. Voice rest after vocal fold surgery: current practice and evidence. J Laryngol Otol 2013; 127: 773-779

[7] Joshi A, Michael M]. Current practices for voice rest recommendations after phonomicrosurgery. Laryngoscope 2018; 128: $1170-1175$
[8] Behrman A, Sulica L. Voice rest after microlaryngoscopy: current opinion and practice. Laryngoscope 2003; 113: 2182-2186

[9] Rousseau B, Cohen SM, Zeller AS et al. Compliance and quality of life in patients on prescribed voice rest. Otolaryngol Head Neck Surg 2011; 144: 104-107

[10] Kaneko M, Hirano S. Voice rest after laryngeal surgery: What's the evidence? Curr Opin Otolaryngol Head Neck Surg 2017; 25: 459-463

[11] Ishikawa K, Thibeault S. Voice rest versus exercise: a review of the literature. J Voice 2010; 24: 379-387

[12] Jette ME, Hayer SD, Thibeault SL. Characterization of human vocal fold fibroblasts derived from chronic scar. Laryngoscope 2013; 123: 738-745

[13] Whitling S, Lyberg-Åhlander V, Rydell R. Absolute or relative voice rest after phonosurgery: a blind randomized prospective clinical trial. Logoped Phoniatr Vocol 2018; 43: 143-154

[14] Kiagiadaki D, Remacle M, Lawson G et al. The effect of voice rest on the outcome of phonosurgery for benign laryngeal lesions: preliminary results of a prospective randomized study. Ann Otol Rhinol Laryngol 2015; 124: 407-412

[15] Fleischer S, Hess M. Postoperativer Stimmgebrauch: Ist Schweigen wirklich Gold? HNO-Nachrichten 2018; 48: 30-35

[16] Titze IR. Voice training and therapy with a semi-occluded vocal tract: rationale and scientific underpinnings. J Speech Lang Hear Res 2006; 49: 448-459

[17] Rubin AD, Praneetvatakul V, Gherson S et al. Laryngeal hyperfunction during whispering: reality or myth? J Voice 2006; 20: 121-127

[18] Hufnagle J, Hufnagle K. Is quiet whisper harmful to the vocal fold mechanism? A research note. Percept Motor Skills 1983; 57: 735-737

[19] Fleischer S, Kothe C, Hess M. Die Kehlkopfkonfiguration beim Flüstern. Laryngo Rhino Otol 2007; 86: 271-275

[20] Sundberg J, Scherer R, Hess $M$ et al. Whispering - a singlesubject study of glottal configuration and aerodynamics. J Voice 2010; 24: 574-584

Bibliografie

DOI https://doi.org/10.1055/a-0949-7218

Online-Publikation: 19.11.2019 | Sprache · Stimme · Gehör 2020; 44: 29-33

(c) Georg Thieme Verlag KG Stuttgart · New York ISSN 0342-0477 